BoRevich, z. I. AND Shafarevich, I. R., Number Theory (Academic Press, New York and London, 1966), $x+435$ pp., 104s.

This is a translation of the original Russian edition, published in Moscow, 1964. The book is not a comprehensive book on Number Theory in the sense of Hardy and Wright; it concentrates on certain selected topics at a level of difficulty considerably above that of Hardy and Wright. The motivating problems that give rise to these topics are diophantine in nature, for example, representation by quadratic forms and Fermat's last theorem. The methods introduced for tackling the problems are developed at some length for their own sakes and include a considerable amount of field theory, valuation theory and in particular $p$-adic techniques, local field theory, the theory of divisors, geometrical lattice theory and various analytic techniques including the use of $p$-adic analytic functions. The work includes a proof of Dirichlet's theorem on prime numbers in arithmetic progression and a proof of Kummer's theorem on the number of divisor classes of a cyclotomic field together with the applications of this result to Fermat's theorem. The combination of motivating problems, development of ideas and techniques and stress on the number theory as a central part of the work has produced an unusual and exciting book, written with considerable style and polish and containing a wide range of important and interesting mathematical results. There are collections of useful problems and interesting tables of facts about quadratic, cubic and cyclotomic fields together with a table listing all irregular prime numbers $\leqq 4001$.

J. HUNTER

MOSTOWSKI, A. AND STARK, M., Introduction to Higher Algebra (Pergamon Press, Oxford, 1964), 474 pp., 45s.

This book is a translation of the original Polish version published by Polish Scientific Publishers in 1958. The bulk of the book is concerned with matrices, determinants, vector spaces, polynomials in one and in several variables and some applications of this range of work. There is also an introduction, presented in three chapters, covering set and function notation, induction, simple combinatorial work and number systems, especially the complex numbers. The emphasis is on technique and the presentation is rather old-fashioned. There is little that can be called abstract and when ideas in this category arise they are treated with a minimum of detail. Nevertheless the book contains a wealth of interesting results and the introductions to most of the topics describe the elementary models generalised in these topics; for example, enough elementary number theory is given to act as a motivating force for the polynomial ring theory that follows.

J. HUNTER

PATTERSON, E. M. AND RUTHERFoRD, D. E., Elementary Abstract Algebra (Oliver and Boyd Ltd., 1965), viii+211 pp., 17s. 6d.

This book contains a useful introduction to many of the topics that are now included in most books and courses on algebra. Whether or not the word abstract should be included in a description of the range of work covered is a matter of taste, but eventually the ideas and techniques involved will be regarded simply as part of necessary basic algebraic tools. These include set notation, equivalence relations, operations on sets, the basic algebraic structures such as semigroups, groups, rings, integral domains, fields, ideals, vector spaces, linear algebras, substructures, factor rings and extensions such as polynomial rings and algebraic extensions of a field. There are many illustrations from and applications in number systems, number theory, euclidean geometry and matrix theory, and the ideas of isomorphism and homomorphism are introduced at elementary level. However there is probably a need 
for more sets of exercises in the book, or for an accompanying exercise booklet, illustrating the rich field of applications of the ideas and listing general results. Another slight criticism that might be made of the overall effect of the book is that too many topics have been introduced into the 200 or so pages with the result that not enough space has been available for motivation and for applications. A professional pure mathematician will not of course feel this lack, but a beginning student or teacher wishing to bring his knowledge up to date may wish for more justification or illustrations at several places in the text.

J. HUNTER

\section{Brief Mentions}

LeITMan, G., Topics in Optimization (Academic Press Inc., New York, 1967), xv+ 469 pp., \$18.00.

This volume includes many results on the optimization of dynamical systems which extend or generalize the findings recorded in Optimization Techniques by the same editor and publisher in 1962. It is divided into two parts of five chapters each, the first describing results obtained by classical variational arguments and the second those involving modern geometrical concepts. The individual chapters coming from twelve authors (including one from Paris and two from Leningrad) are well written and the editor, who is also one of the contributors, has done a good job in producing a review of recent progress in a subject of increasing practical importance and theoretical interest.

KUSHNer, H. J., Stochastic Stability and Control (Academic Press Inc., New York, 1967), $x i+161 \mathrm{pp}$.

This monograph is devoted to the study of qualitative properties of Markov processes by the stochastic Liapunov function approach. The first two chapters treat respectively the necessary basic concepts from probability theory and stochastic stability. The remaining chapters are concerned with applications to the study of first exit times, of problems in optimal control, and of the design of optimal controls. It contains much original material and is well written so can be strongly recommended to applied mathematicians, statisticians and automatic control engineers.

TULlER, A., A Modern Introduction to Geometries (D. van Nostrand \& Co., London, 1967), ix+201 pp., 60s.

This compact book (suitable for undergraduates) illustrates the two principal approaches to geometry: the development of a body of theorems from a set of axioms and the study of the invariant theory of a transformation group. It is clearly written and attractively produced.

wallace, A. W., Differential Topology: First Steps (W. A. Benjamin Inc., New York, $1968), 130$ pp., $\$ 9.50$ cloth, $\$ 3.95$ paper.

Written for students with no previous knowledge of topology this small book is a useful introduction to certain aspects of the topology of manifolds which can be discussed without the use of advanced algebraic methods. The method of spherical modifications and the study of critical points of functions on manifolds are clearly presented. The emphasis throughout is on the geometric meaning of the ideas under consideration and on the motivation of the abstract concepts introduced. 